Ein verhältnismäßig geringer mathematischer Aufwand wurde durch eine mit den Mitteln der Theorie der Zufallsfunktionen bewerkstelligten Abstraktion von den individuellen Zügen spezieller Geschwindigkeitsfelder erreicht.

Gerade die Abstraktion von den individuellen Eigenschaften läßt eine gemeinsame Eigenschaft dieser Felder deutlich hervortreten, die anscheinend für die Wirksamkeit als Dynamo notwendig ist: Die Auszeichnung eines Schraubensinnes.

Das innere Produkt $\mathfrak{b}$ rot $\mathfrak{b}$ ist auf Grund der Definition von rot $\mathfrak{b}$ offensichtlich ein $\mathrm{Ma} \beta$ dafür, ob ein Schraubensinn und welcher Schraubensinn durch das Feld $\mathfrak{b}$ ausgezeichnet wird.
Es wird also der Schluß nahegelegt, daß ein definites Vorzeichen des inneren Produktes $\mathfrak{b} \cdot \operatorname{rot} \mathfrak{b}$ in räumlich ausgedehnten Gebieten eine notwendige Bedingung dafür ist, daß das Geschwindigkeitsfeld $\mathfrak{v}$ Dynamowirkung zeigt.

Die Bedingung $\mathfrak{v} \cdot \operatorname{rot} \mathfrak{b}=0$ ist charakteristisch für flächennormale Felder. Die Aussage, daß von flächennormalen Geschwindigkeitsfeldern keine Dynamowirkung ausgeht, könnte eine Präzisierung des Theorems von Cowling ${ }^{11}$ sein; ein Beweis dieser Aussage liegt allerdings bisher nicht vor.

11 T. G. Cowling, Monthly Notices Roy. Astron. Soc. 94, 39 [1934].

\title{
Angular Dependence of Channelling in Gold Single Crystals
}

\author{
R. D. Schuckert, \\ Physik-Department der Technischen Hochschule München \\ H. Lutz, \\ Oak Ridge National Laboratory, Tennessee, USA \\ and R. SizmanN \\ III. Physikalisches Institut der Universität München \\ (Z. Naturforschg. 21 a, 1296-1299 [1966] ; received 2 April 1966)
}

\begin{abstract}
The penetration profiles of $70 \mathrm{keV}$ krypton-85 ions in gold single crystals were determined for different incident angles between the ion beam and the $\langle 110\rangle$ or $\langle 111\rangle$ crystal axis. Channelling is most pronounced in the 110 direction but it falls off at first steeply, then at angular deviations larger than $10^{\circ}$ rather slowly by tilting to $\langle 111\rangle$ or $\langle 100\rangle$. A sharp aperture cannot be defined for the 110 channel; its half width is of the order of $2 \ldots 3^{\circ}$. In $\langle 111\rangle$ no directional channel seems to be operative, although the long tail range distribution indicates a steering of the penetrating ions.
\end{abstract}

The strong influence of crystallographic orientation on the penetration depth of fast ions in solids has been shown theoretically and experimentally ${ }^{1}$. A particle, penetrating along certain low index directions (e.g. $\langle 100\rangle$ and $\langle 110\rangle$ in fcc metals) is subject to reflective forces from the neighbouring atomic rows which tend to keep it moving straight ahead. The steering leads to ranges much farther than the usual mean range. This channelling, as it

1 M. T. Robinson and O. S. Oen, Bull. Am. Phys Soc. 7, 171 [1962] ; Appl. Phys. Letters 2, 30 [1963] ; Phys Rev. 132, 2385 [1963]. - G. R. Pierey, F. Brown, J. A. Davies, and M. McCargo, Phys. Rev. Letters 10, 399 [1963]. - H. Lutz and R. Sizmann, Phys. Letters 5, 113 [1963]. - R. S. Nelson and M. W. Thompson, Phil. Mag. 8, 1677 [1963]. - C. LeHmann and G. Leibfried, J. Appl. Phys. 34, 2821 [1963]. J. Lindhard, Phys. Letters 12, 126 [1964] ; Kgl. Danske Videnskab. Selskab, Mat. Fys. Medd. 34, No. 14 [1965]. was called, occurs readily when bombarding a perfect single crystal with an ion beam parallel to an open lattice direction. However, the yield of far penetrating ions depends critically on the angle of the impinging particles with respect to the favourable crystallographic axis. Hitherto, this angular dependence of channelling has been investigated for light projectiles (protons, $\alpha$-particles) in the energy range between $27 \mathrm{keV}$ and $7.7 \mathrm{MeV}^{2}$ and for rather

2 W. Brandt, J. M. Khan, D. L. Potter, R. D. Worley, and H. P. Sмiтн, Phys. Rev. Letters 14, 42 [1965]. - M. W. Thompson, Phys. Rev. Letters 13, 756 [1965]. - E. Вøgн, J. A. Davies, and K. O. Nielsen, Phys. Letters 12, 129 [1964]. - D. S. Gemmel and R. E. Holland, Phys Rev. Letters 14, 945 [1965]. - R. S. Nelson and M. W. Thompson, Phil. Mag. 8, 1677 [1963]. - B. Domeis and K. BлörKQuist, Phys. Letters 14, 127 [1965]. 
highly energetic heavy ions in the region 20 to $100 \mathrm{MeV}^{3}$. In both cases the angular width of channelling in several fec metals is found to be smaller than a few degrees.

We have now investigated in more detail the penetration behaviour of a heavy particle, krypton 85 , with the moderate energy of $70 \mathrm{keV}$ in gold single crystals. In contrast to the quoted experiments the steering forces of the atomic rows in this case are believed to be due to much more strongly shielded atomic interactions with considerable overlap of the repulsive potentials between the rows.

\section{Experimental}

The applied range measurement technique corresponds in principle with the details given in ${ }^{4}:$ a parallel beam of $\mathrm{Kr}-85$ ions from a linear accelerator set at $70 \mathrm{keV}$ hits the gold crystal whose crystallographic axis has a known orientation to the beam direction. After the bombardment the radioactivity of the crystal is determined. The count rate is assumed to be proportional to the total number of $\mathrm{Kr}-85$ atoms which have penetrated into the crystal. By applying low energy $(3 \mathrm{keV})$ sputtering with Xe-ions, thin layers ( $20 \AA$ or more) of the gold crystal are removed in a controlled manner. The decrease in count rate is measured successively. From the known sputtering ratio the integral range distribution can be plotted as a function of penetration depth.

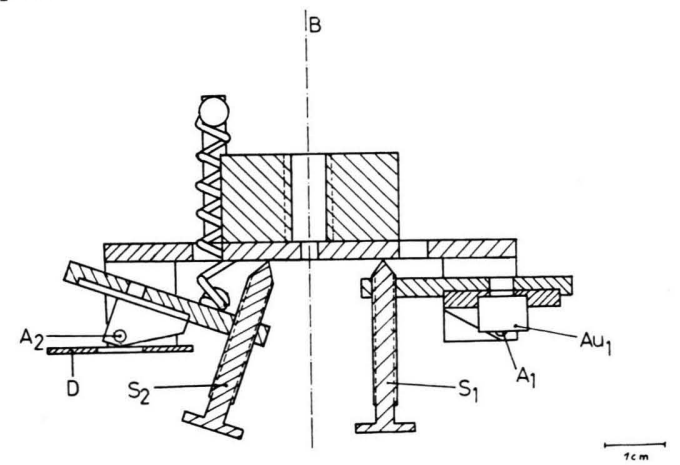

Fig. 1. Crystal holder with two gold single crystals $\mathrm{Au}_{1}$ and $\mathrm{Au}_{2}$ (not shown), turnable about the axes $\mathrm{A}_{1}$ and $\mathrm{A}_{2}$, respectively. $S_{1}$ and $S_{2}$ fine pitch thread screws. D, diaphragm. The axis $B$ is parallel to the beam direction and perpendicular to $A_{1}$ and $A_{2}$.

3 S. Datz, T. S. Noggle, and C. D. Moak, Nucl Instr. Methods 38, 221 [1965]. - O. Selig and R. Sizmann, Nukleonik, im Druck [1966].
To match the presumed strong dependence of channelling on crystal orientation, an accurate adjustment of the gold crystals with regard to the beam direction has been necessary. Fig. 1 shows the device used throughout the experiments. Two gold crystals $\mathrm{Au}_{1}$ and $\mathrm{Au}_{2}$ with respectively (110) and (111) surface orientation are fixed in a holder. Each crystal can be tilted separately about the axis A by turning the fine pitch thread screws $S_{1}$ and $S_{2}$, respectively. The holder is turnable about axis $\mathrm{B}$ to allow each crystal to be alternatively brought into the Kr-85 beam or, afterwards, into the sputtering or counting positions. The reproducibility of the settings in $\mathrm{A}$ axis has been shown by optical means to be better than $4^{\prime}$.

Special care has been taken to prepare the crystal surfaces. Firstly, they were electropolished in a mixture of 1 part $\mathrm{HNO}_{3}$ with 3 parts of $\mathrm{HCl}, \mathrm{H}_{3} \mathrm{PO}_{4}$, acetic acid, and water each. Then they were sputtered half an hour with an intense beam of $2 \mathrm{keV}$ nitrogen ions (approximately $5 \mathrm{~mA} / \mathrm{cm}^{2}$ ) for the further cleaning and smoothing of the surfaces.

The crystals were tightly fixed in the holders and then their orientation was determined by LAUE back reflection photography. The two crystals were progressively adjusted so that ultimately the 110- and 111-axis, respectively, were exactly parallel to the ion beam direction with the corresponding 100 and 110 in the tilting axes A of Fig. 1. A possibly remaining deviation would have been smaller than $0.5^{\circ}$. Further more, the zero setting of the angle $\alpha$ between the 110 crystal axis and beam direction was checked experimentally and it corresponded with the highest yield in channelled ions. The reproducibility of different angles $\alpha$, very close to $\alpha=0^{\circ}$ was checked by several independent range measurements and found to be satisfactory.

After the final adjustments the crystals were sputtered with $3 \cdot \mathrm{keV}$ Xe-ions up to a total dose of $10^{17} / \mathrm{cm}^{2}$. Then they were turned to a certain angle $\alpha$, bombarded with $\mathrm{Kr}-85$, turned back to $\alpha=0$ and sputtered with Xe-ions to determine the range distribution. Thus, sputtering was always done with normal incidence of the Xe-ions to the crystal surface.

The calibration of the sputtering time (at constant current density) into Ångstrøm was done for

${ }^{4}$ H. Lutz and R. Sizmann, Z. Naturforschg. 19 a, 1079 [1964]. 
the 111-gold face by determining the weight loss of the crystal after several high Xe-ion-doses. We found a sputtering ratio of $S_{111}=12 \pm 1.5$ gold atoms/XeIon. The sputtering ratio of the 110 gold surface was determined from a comparison of the ranges in the two crystals: both the (111) and the (110) crystals were tilted through nearly $17^{\circ}$ to expose the same crystalline orientation to the ion beam direction. Hence, in this position the Kr-85 range distributions in both crystals are the same. Since the sputtering is done on two different surface orientations, 111 and 110, respectively, the relative sputtering ratios $S$ can be fixed. The result is $S_{111}: S_{110}=2: 1$ within an experimental error of $\pm 7 \%$.

Finally we have to consider the angular divergence of the Kr-85 beam. Fig. 2 shows the beam profile as determined from autoradiographs by placing diaphragms at different positions in the track of the ion beam. The half width is ca. $1^{\circ}$, a rather undesirable feature of our accelerator.

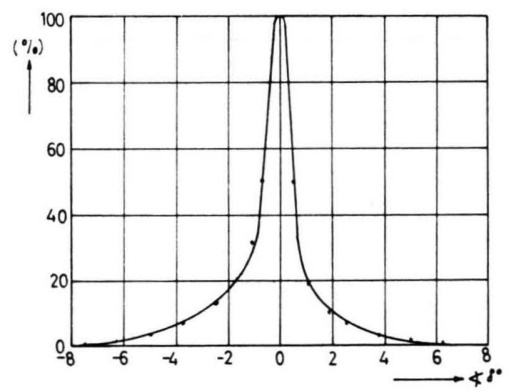

Fig. 2. Angular divergence of the incident $\mathrm{Kr}-85$ beam.

\section{Results}

Three series of range measurements were taken:

(i) The ion beam incident in the 110 direction and stepwise turning to the 111 axis.

(ii) The ion beam incident in the 110 direction and stepwise turning to the 100 axis.

(iii) The ion beam incident in the 111 direction and stepwise tilting to the 110 axis.

Fig. 3 a shows the combined results of the series (i) and (iii) ; Fig. $3 \mathrm{~b}$ shows the ranges of the series (ii). The angle notation $\alpha$ always refers to the beam direction with respect to the 110 axis. In the case of series (i) we have tilted to both sides of the 110 axis $(\langle 110\rangle \rightarrow\langle 111\rangle$ and $\langle 110\rangle \rightarrow\langle 11 T\rangle)$.
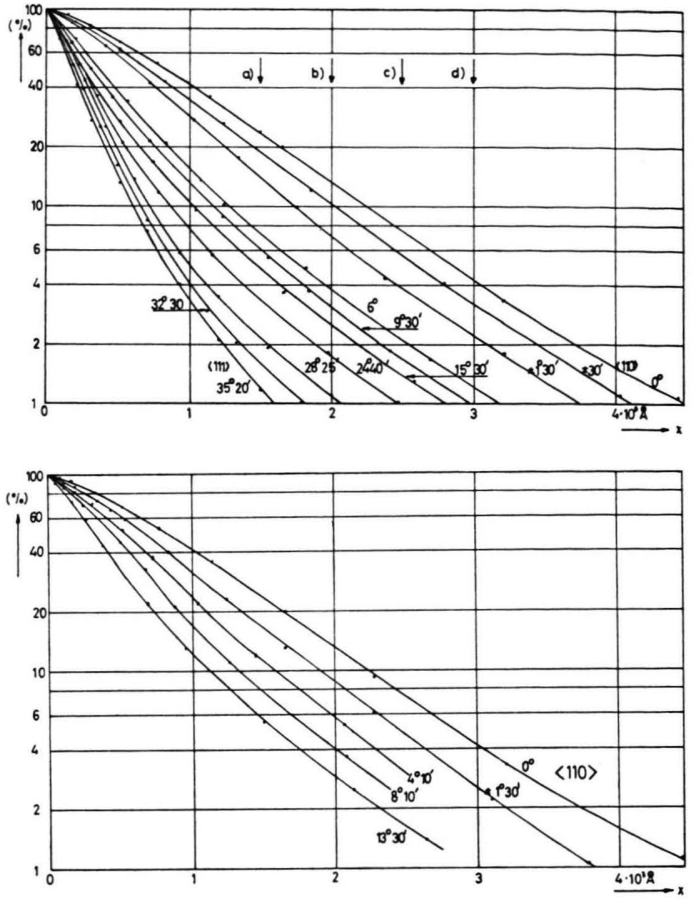

Fig. 3. Range measurements of $70 \mathrm{keV} \mathrm{Kr}-85$ ions in gold single crystals. The ordinate gives the residual radioactivity of the bombarded crystal, the abscissa the thickness in $10^{3} \AA$ of the gold layer removed by sputtering. a) Angular dependence of the penetration by turning from $\langle 110\rangle$ into $\langle 111\rangle$ with the ion beam remaining in the 110 plane. The positions marked a, b, c, and d correspond to the plots in Fig. 4. b) Angular dependence of the penetration by turning from $\langle 110\rangle$ into $\langle 100\rangle$ with the beam remaining in the 100 plane.

The range distributions with equal deflections $\alpha$ coincide completely as can be seen in Fig. 3 a. This proves that at the setting $\alpha=0$ the ion beam is exactly parallel to the crystal axis. In the series (i) and (ii) the ranges with $\alpha=0$ do also coincide. Both curves are measured independently since the positions of the gold crystal with respect to the tilting axis $\mathrm{A}$ of the crystal holder are different (the A axis is parallel to $\langle 110\rangle$ in the case (i) and parallel to $\langle 001\rangle$ in the case (ii)).

The range distributions in Fig. $3 \mathrm{a}, \mathrm{b}$ are the original experimental results. They were not corrected for the oblique incidence of the ion beam at the different angles $\alpha$. Although in Fig. 3 a the parameter $\alpha$ amounts to $35.3^{\circ}$ (the angle between the 111 and the 110 axis) in the experiments each crystal has been tilted only through ca. $17^{\circ}$. Therefore, a correction factor $1 / \cos \alpha$ would effect the measured penetration depth less than 4.5\%. "Normal" range distributions should obviously be corrected 
with $1 / \cos \alpha$ but it would be a rather arbitrary correction procedure for range profiles with channelling distributions in fixed crystalline directions.

\section{Discussion}

The channelling can be seen best from the tails of the range curves in Fig. 3 a, b. It may be assumed that beyond a depth of three times the most probable range only channelled krypton ions are contributing to the penetration. Therefore, we have plotted for several abscissa values $x \geqq 1,500 \AA$ the dependence of the residual krypton content vs. the incident angle $\alpha$, Fig. 4 . From the Figs. 3 and 4 the following implications can be drawn:

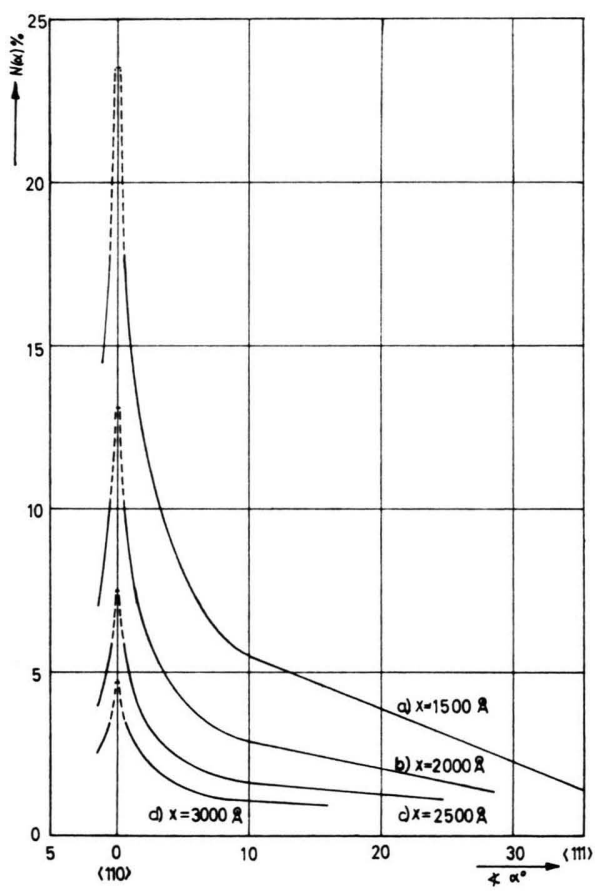

Fig. 4. Distribution of residual activity as a function of beam direction $\alpha$ with respect to the 110 crystal axis, turning from $\langle 110\rangle$ into $\langle 111\rangle$ in the plane 110 . The parameters a ...d correspond to the increasing penetration depths of Fig. 3 a.

(i) the angular dependence of channelling in the 110 direction is different for tilting to $\langle 100\rangle$ or $\langle 111\rangle$; to the 111 direction the channel seems to be narrower. This is to be expected also from the geometry of atomic positions in the fcc lattice. (ii) the variation of the fraction of channelled krypton ions with $\alpha$ is steeper for the greater depths of the range curves.

Although the divergence of the primary ion beam has to be considered, its influence can not fully account for this behaviour.

(iii) a characteristic aperture of the 110 channel direction cannot be stated unambiguously. With $x>2500 \AA$ a value of $2 \ldots 3^{\circ}$ seems to be appropriate, independent of the tilting from $\langle 110\rangle$ to $\langle 111\rangle$ or $\langle 100\rangle$.

(iv) a remarkable feature appears to be the behaviour of the 111 direction with variation of $\alpha$. In contrast to the maximum of penetration observed exactly in the 110 axis the penetration in the 111 direction has a minimum. We have to conclude that the $\langle 111\rangle$ direction per se is not effective for channelling of $70 \mathrm{keV}$-krypton ions; at least an $\langle 111\rangle$ channel cannot have an aperture greater than $1^{\circ}$.

In fact 111 planes are much more closely packed than $\{110\}$ or $\{100\}$; from this it may be concluded that fast ions impinging on $\{111\}$ are efficaciously deflected into other directions. However, the range curve of the 111 direction in Fig. $3 \mathrm{a}$ is certainly different in shape from a pure random scattering process; there is an approximately $4 \%$ contribution of far penetrating ions. Also Monte Carlo calculations with a high speed computer ${ }^{5}$ suggest, that some of the krypton ions incident in $\langle 111\rangle$ are subjected to steering, possibly between 110 planes. A further support for such a planar channelling may be seen in the previously unexpected slow decrease of the long range tail for angular deviations $\alpha>10^{\circ}$ off the 110 direction. One has at least to be careful in comparing experimental ranges with theoretical considerations of the penetration along a fixed axis. In the usual techniques of range measurements in thick crystals only the projection of the true range and the range straggling upon the direction of investigation can be found. However, further explicit experimental evidence is needed for the efficacy of planar effects in the penetration behaviour of heavy ions of moderate kinetic energy in close packed crystal structures.

5 H. Lutz, R. Schuckert, and R. Sizmann, Nucl. Instr. Methods 38, 241 [1965]. 